\title{
TANNENBERG (GRUNWALD) IN DER DEUTSCHEN TRADITION DES 19. UND 20. JAHRHUNDERTS
}

\author{
KEYWORDS
}

Teutonic Order; Tannenberg (Grunwald); 1410; 1914; Historical Symbols; School Books

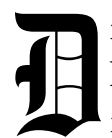
ie Schlacht bei Tannenberg war für den Deutschen Orden eine katastrophale Niederlage. Sein Glück bestand darin, dass die polnisch-litauische Belagerung des Hochmeistersitzes Marienburg erst mit Verzögerung begann. Das gab dem Komtur von Schwetz Heinrich von Plauen die notwendige Zeit, alle noch vorhandenen Kräfte auf die Belagerung vorzubereiten. Der mangelnde Erfolg der Belagerung verschaffte dem Orden in Preußen die Überlebensmöglichkeit, auch wenn die im Thorner Frieden von 1411 vereinbarten Zahlungen ihn hart trafen. Auf polnischer Seite wurde das Kriegsziel, die Gewinnung Pommerellens, nicht erreicht, der Orden konnte sein Territorium halten. Das milderte den Eindruck, der Orden sei der katastrophale Verlierer. Doch die Kenntnis von der verlorenen Schlacht besaß eine Eigendynamik. Man wusste davon im nahegelegenen Lübeck ebenso wie in Osnabrück, Braunschweig und Magdeburg, in Augsburg und in Bayern, im österreichischen Melk wie in Klosterneuburg bei Wien, in Schlesien wie in Böhmen und in Frankreich ${ }^{1}$; siebzig Jahre später nahm der Schweizer Diebold Schilling eine prächtige Schlachtenzeichnung in seine Spiezer Bilderchronik und 1513 eine andere in seine Luzerner Chronik auf.2 Ganz Europa erfuhr von

1 Vgl. die Quellenauszüge in: Scriptores rerum Prussicarum. Die Geschichtsquellen der preußischen Vorzeit bis zum Untergange der Ordensherrschaft, hrsg. v. T. Hirsch, M. Töppen, E. Strehlke, Bd. III, Leipzig 1866 (Nachdr. Frankfurt/Main 1965), S. 405-461. Vgl. auch M. Biskup, Echa bitwy grunwaldzkiej i oblężenia Malborka w niemieckiej gatęzi Zakonu Krzyżackiego w lecie 1410 roku, Komunikaty Mazursko-Warmińskie, 1983, 4 (162), S. 455-460.

2 Diebold Schilling, Spiezer Bilderchronik, Luzern 1484/85, hrsg. v. H. Haeberli, V. Bartlomé, Luzern 1991; vgl. auch Idee Europa. Entwürfe zum „Ewigen Frieden“. Ordnungen und Utopien für die Ge- 
der Niederlage des Ordens. Damit war der Deutsche Orden ideologisch verletzbar geworden, wie sich auf dem Konzil von Konstanz wenige Jahre später zeigte. Er hatte nicht nur eine Schlacht verloren, sondern auch den Nimbus der Unbesiegbarkeit - Tannenberg galt nunmehr als Schmach, die wie eine Hypothek auf der Ordensgeschichte lastete. Wie konnte es dazu kommen, dass die Niederlage Jahrhunderte später einen solch hohen Stellenwert im Bewusstsein erlangte, geradezu zum Symbol wurde?

Nach dem Verlust Preußens und Livlands im 16. Jahrhundert wie auch der Besitzungen im Mittelmeerraum hatte der Orden nur noch im Heiligen Römischen Reich deutscher Nation überlebt. Napoleon zerschlug dieses Reich, damit 1809 auch die Existenz des Ordens auf deutschem Boden. Der Orden existierte nur noch in der Habsburgermonarchie. Dort erfuhr er Reformen und Neuansätze, die ihn heute in sieben europäischen Staaten als priesterlich-karitative Gemeinschaft der katholischen Kirche wirken lassen. ${ }^{3}$

Die Zerschlagung des Heiligen Römischen Reichs deutscher Nation bedeutete gleichzeitig die Niederlage des preußischen Königreichs. Erst Napoleons Katastrophe von Moskau brachte den Umschwung. Die anschließenden deutschen Befreiungskriege gingen von Preußen aus, in ihnen wurde erstmals auf die Tradition des mittelalterlichen Deutschen Ordens in Preußen zurückgegriffen. Denn nachdem Napoleon den Orden enteignet und vertrieben hatte, stand diesem Rückgriff nichts mehr im Wege.

Diese Traditionsaneignung begann mit der Stiftung des Eisernen Kreuzes 1813 als preußische Kriegsauszeichnung (Abb. 1). ${ }^{4}$ Sie wurde weitergeführt in der Lite-

staltung Europas von der pax romana zur Europäischen Union (Katalog), hrsg. v. M.-L. v. Plessen, Berlin 2003, S. 82. Zu beiden Darstellungen vgl. ausführlich mit weiteren Beispielen und Abbildungen K. Sroczynska, Ze studiów nad ikonografia bitwy pod Grunwaldem, Rocznik Olsztyński 4 (1961/1962), S. 53-104; zuletzt mit weiterer Literatur U. Arnold, Zobrazováni bitvy u Grunwaldu. Die Schlacht von Tannenberg im Bild, in: Svet tajemnych Baltu. The World of the Mysterious Balts, hrsg. v. L. Jan, P. Kosthun, Z. Nerudová, Brno 2013, S. 159-169 (tschechisch/deutsch).

3 Eine ausführliche wissenschaftliche Gesamtdarstellung seiner Geschichte bis in die Gegenwart fehlt; den besten Überblick bieten M. Tumler, U. Arnold, Der Deutsche Orden. Von seinem Ursprung bis zur Gegenwart, Bad Münstereifel ${ }^{5} 1992$; U. Arnold, L'Ordine Teutonico - una viva realtà, Lana 2001.

4 Vgl. zum Folgenden H. Boockmann, Der Deutsche Orden. Zwölf Kapitel aus seiner Geschichte, München 1981, ${ }^{5}$ 1999, S. 234-254; ders., Zakon Krzyżacki. Dwanaście rozdziatów jego historii, übers. v. R. Traba (Klio w Niemczech 3), Warszawa 1998; 800 Jahre Deutscher Orden. Ausstellung des Germanischen Nationalmuseums Nürnberg in Zusammenarbeit mit der Internationalen Historischen Kommission zur Erforschung des Deutschen Ordens (Katalog), hrsg. v. G. Bott, U. Arnold, Gütersloh-München 1990, S. 437-505; U. Arnold, Nationalismus, Nationalsozialismus und der Mißbrauch der Deutschordenstradition in Deutschland, in: Der Deutsche Orden und die Ballei ElsaßBurgund. Die Freiburger Vorträge zur 800-Jahr-Feier des Deutschen Ordens, hrsg. v. H. Brommer, 
ratur, in der Malerei, in der Historiographie - letztlich wurde sie Gemeingut des öffentlichen Bewusstseins in Preußen und ab 1871 im neuen, preußisch geführten Deutschen Reich. Der Ordensstaat galt als Keimzelle des Preußentums, des Deutschtums, sein Kreuz überlebte im militärischen Bereich alle Regierungsumbrüche von 1813 bis in die Gegenwart der Bundesrepublik. Der Ordensstaat galt im allgemeinen Bewusstsein als deutschester aller deutschen Staaten, seine mittelalterliche Multiethnizität wurde umgedeutet zur erfolgreichen Assimilation von Prußen und Slawen unter deutscher Führung. Der Ordensritter stieg auf zum Symbol des deutschen Helden schlechthin, gleichgestellt mit dem christlichen Retter St. Georg ebenso wie mit dem Reichspatron St. Michael (Abb. 2). ${ }^{5}$

Das Königreich Preußen war eine der Mächte gewesen, die Polen im 18. Jahrhundert geteilt hatten. Diese Teilung dauerte auch im 19. Jahrhundert an, während Preußen nach seiner tiefen Erniedrigung unter Napoleon den Aufstieg zur führenden Macht des europäischen Festlandes vollzog. Die preußische positive Traditionsaneignung des mittelalterlichen Ordensstaates rief daher in Polen eine Gegenbewegung hervor. Der strahlende Ordensritter auf preußisch-deutscher Seite wurde in Polen ebenso zum Symbol, zum verhassten, blutrünstigen krzyzak. Auch das vollzog sich in der Literatur, in der Malerei, in der Historiographie, bis zu Denkmälern und öffentlichen Gedenkfeiern. Diese negative Form der Traditionsaneignung ging gleichermaßen in das polnische allgemeine öffentliche Bewusstsein ein wie die positive in Deutschland.

Die historische Entwicklung des Ordens in Preußen war jedoch keine geradlinige Erfolgsgeschichte gewesen. Sie besaß zwei entscheidende Bruchstellen, das Ende 1525 und die Schlacht von Tannenberg 1410. Mit 1525 konnte auch die deutsche Seite im 19. Jahrhundert gut leben. Schließlich bedeutete der Eid von Krakau durch

Bühl-Baden 1996, S. 205-222; ders., Der Deutsche Orden im deutschen Bewußtsein des 20. Jabrhunderts, in: Vergangenheit und Gegenwart der Ritterorden. Die Rezeption der Idee und die Wirklichkeit, hrsg. v. Z. H. Nowak, R. Czaja (Ordines Militares. Colloquia Torunensia Historica XI), Toruń 2001, S. 39-53, Wiederabdruck in: U. Arnold, Deutscher Orden und Preußenland. Ausgewäblte Aufsätze anläßlich des 65. Geburtstages, hrsg. v. B. Jähnig, G. Michels (Einzelschriften der Historischen Kommission für ost- und westpreußische Landesforschung 26), Marburg 2005, S. 93-106; ders., Die Marienburg alspolitisches Symbol in Deutschland in der ersten Hälfte des 20. Jahrbunderts, in: Praeterita posteritati. Studia z historii sztuki i kultury ofiarowane Maciejowi Kilarskiemu, hrsg. v. M. Mierzwinski, Malbork 2001, S. 33-40, polnisch: Zamek w Malborku jako symbol polityczny Niemiec w pierwszejpolowie XX wieku, in: ebd., S. 41-44; ders., Tannenberg/Grunwald alspolitisches Symbol im 19./20. Jabrhundert, in: Krajobraz grunwaldzki w dziejach polsko-krzyżackich i polskoniemieckich na przestrzeni wieków. Wokót mitów i rzeczywistości, hrsg. v. J. Gancewski (Biblioteka „Magrowskich Studiów Humanistycznych”, Historia 1), Olsztyn 2009, S. 7-18; weißrussische Fassung (mit Nachtrag zu 2010): Belarusian Historical Review 2011, S. 146-166. Abb. des Eisernen Kreuzes von 1813 in: 800 Jahre (wie oben), S. 448.

5 Abbildung in: 800 Jahre (wie Anm. 4), S. 475. 
den Hochmeister und künftigen Herzog in Preußen Albrecht von BrandenburgAnsbach den Beginn der Hohenzollern in einem Gebiet, das knapp 200 Jahre später der neuen Monarchie sogar den Namen gab. Für 1410 sah dies jedoch anders aus.

Bei der Identifikation mit dem Deutschen Orden in Preußen/Deutschland empfand man die Schlacht als Schmach ${ }^{6}$, als fortdauernde Niederlage. Tannenberg war für die preußisch/deutsche Seite nichts, was man hätte feiern können. Wie also ging man damit um? Am stärksten meinungs- und bewusstseinsbildend für alle Bevölkerungsschichten war im 19. Jahrhundert die Schule. Zum einen hatte der Geschichtsunterricht innerhalb des Fächerkanons noch eine weit größere Bedeutung als heute, zum zweiten gab es den Einfluss vieler Medien - wie etwa des Fernsehens - noch gar nicht oder er war schon quantitativ viel geringer - wie etwa des Buches als Unterhaltungslektüre. Deshalb habe ich zur Beantwortung meiner Frage einige Schulbücher des 19. Jahrhunderts gegriffen.7 In Geschichtlicher Leitfaden für Anfänger, 1875 im preußischen Rheinland, also weit im Westen der Monarchie erschienen, gibt es für die Ordensgeschichte drei Jahreszahlen: 1309 Marienburg wird Hochmeistersitz, 1466Zweiter Thorner Frieden, 1525 Umwandlung des Ordenslandes in ein weltliches Herzogtum ${ }^{8}$ - Tannenberg kommt nicht vor. Etwas älter ist die Kleine Vaterländische Geschichte. Ein Leitfaden für preußische Volksschulen, vielleicht erstmals in den 60er Jahren im preußischen Halle an der Saale erschienen. Das Buch war sehr beliebt mit einer hohen Auflagenzahl. Für die Ordensgeschichte wird der Bau der Marienburg erwähnt ${ }^{9}$, mehr nicht; auch in der Zeittafel ${ }^{10}$ fehlt Tannenberg. Das war e in e Form des Umgangs mit der Niederlage von 1410 - Verschweigen. Damit ergab sich offenbar eine Fortsetzung der Situation des 18. Jahrhunderts, von der Sven Ekdahl sagt: „Zu dieser Zeit war ,Tannenberg' in Preußen eben kein Thema mit symbolischer Bedeutung ". ${ }^{11}$

Eine andere Form zeigt die nächste Gruppe der Schulbücher. Bereits 1832 oder 1833 erschien in Potsdam der Grundriß der Weltgeschichte für Gymnasien, höhere Lehranstalten und zum Selbstunterricht: Die große Niederlage bei Tannen-

6 Dieser Begriff auch benutzt von A. Kossert, Ostpreußen. Geschichte und Mythos, Berlin ${ }^{32} 2005$, S. 48.

7 Die exemplarische Auswahl geschah unter dem Aspekt möglichst weiter Verbreitung in Preußen, da die Sichtweise dort bereits vor der Reichsgründung 1871 grundgelegt und anschließend fortgesetzt wurde; vgl. die in Anm. 4 genannte Literatur, deren Ergebnisse hier nicht wiederholt, sondern ergänzt werden sollen. Die Zahl der untersuchten Bücher könnte ohne Weiteres vermehrt werden, was das Ergebnis jedoch nicht wesentlich beeinflussen würde.

8 J. C. Andrä, Geschichtlicher Leitfaden für Anfänger, Bad Kreuznach 1875, S. 93.

9 A. Hummel, Kleine vaterländische Geschichte. Ein Leitfaden für preußische Volksschulen, Halle, hier benutzt ${ }^{27} 1887$, S. 17.

10 Ebd., S. 59 f.

11 S. Ekdahl, Tannenberg/Grunwald - ein politisches Symbol in Deutschland und Polen, Journal of Baltic Studies 22 (1991), S. 271-324, hier S. 276. 
berg (1410) gegen Wladislaw II. von Polen brach die Macht des Ordens auf immer; nur die tapfere Vertheidigung Marienburg's durch den Komthur (bald Hochmeister) Heinrich Reuß von Plauen rettete ihn vor dem Untergange. ${ }^{12}$ Auch im sehr beliebten Grundriß der brandenburgisch-preußischen Geschichte, 1868, also kurz vor der Reichsgründung erstmals erschienen, findet sich dieses Faktenpaar Niederlage bei Tannenberg - Rettung der Marienburg. ${ }^{13}$ In der komprimierten Form eines Repetitoriums wird diese Gegenüberstellung besonders deutlich. Unter der Sparte Fürsten- und Ländergeschichte ist zu lesen: 1410 Niederlage der Deutschritter bei Tannenberg durch Wladislaus Jagiello, Grossfürsten von Litauen/König von Polen [...]. Heinrich v. Plauen rettet die Marienburg. ${ }^{14}$ Das können wir weiter verfolgen bis in das Buch Mit Gott für Kaiser und Reich! Volkstümliche Darstellung der Preußischdeutschen Geschichte, gerade vor der Jahrhundertwende erschienen im ebenfalls zum preußischen Königreich gehörenden Breslau. ${ }^{15}$ Das war die zweite Form, mit der Niederlage von Tannenberg umzugehen: Man stellte ihr sofort die Rettung der Marienburg und damit des Ordens an die Seite mit einem Frieden, dessen Bedingungen [...] für den Orden noch günstig waren. ${ }^{16}$ Man wandelte die Niederlage damit fast in einen Erfolg um. Es muss allerdings betont werden, dass dies die preußische Sicht war. Eine 1855 erschienene Weltgeschichte für höhere Schulen und Selbstunterricht sieht es deutlich anders: Der Sieg der Polen bei Tannenberg im Jahre 1410 brach die Kraft des Ordens und mit dem Unglück von Außen begannen Zwietracht und Entartung. Somogitien und die südöstliche Landschaft Preußens, Sudauen, waren der Preis eines im Jahre 1422 geschlossenen Friedens. ${ }^{17}$ Keine Heldentat der Rettung Marienburgs, sofort der Sprung zum Frieden vom Melnosee unter Auslassung des Thorner Friedens und weiterer zwei Waffengänge. Bezeichnenderweise erschien das Buch nicht in Preußen, sondern in Baden in dem katholischen Verlag Herder.

Ein weiteres Genre neben Schulbüchern sind die Reise - und Unterhaltungsschriftsteller, die vor allem vom gehobenen Bürgertum und dem Adel gelesen wurden. Neben Erzählungen über ferne Länder und Abenteuer, wie etwa von Karl May oder - in Übersetzung - von James Fenimore Cooper, traten in reicher

12 E. A. Schmidt, Grundriß der Weltgeschichte für Gymnasien, höhere Lehranstalten und zum Selbstunterricht, Zweiter Theil: Geschichte des Mittelalters, Potsdam, hier benutzt ${ }^{6} 1851$, S. 132.

13 K. Tücking, Grundriß der brandenburgisch-preußischen Geschichte, Paderborn, hier benutzt ${ }^{3} 1901$, S. 30 .

14 F. Junge, Geschichtsrepetitionen für die oberen Klassen höherer Lehranstalten, Berlin 1885, hier benutzt ${ }^{2} 1893$, S. $72 \mathrm{f}$.

15 M. Haekel, Mit Gott für Kaiser und Reich! Volkstümliche Darstellung der Preußisch-deutschen Geschichte, bearbeitet für Schule, Heer und Haus, Breslau 1899, S. 30 f.

16 Ebd., S. 31.

17 K. Kiesel, Die Weltgeschichte für höhere Schulen und Selbstunterricht übersichtlich dargestellt, Zweiter Band: Die christliche Zeit, Erste Abtheilung: Fünfzehn Jahrbunderte, Freiburg i. B. 1855, S. 460. 
Zahl auch historische Darstellungen. Besonders beliebt war Ritter- und Ritterordensliteratur. Eine interessante, frühe Stimme ist Carl Julius Weber. Er stammte aus Hohenlohe in Franken, nicht aus Preußen. 1792 bis zu dessen Tod 1799 war er Privatsekretär und Regierungsrat in Diensten des Reichsgrafen Christian zu ErbachSchönberg. ${ }^{18}$ Der Deutschordensritter Erbach residierte von 1783 bis zu seinem Tod 1799 als Statthalter des Deutschen Ordens, also Ranghöchster nach dem Hochmeister, in Mergentheim, der damaligen Ordenszentrale. ${ }^{19} 1824$ erschien der 3. Band von Webers Darstellung über das Ritterwesen und die drei großen Ritterorden ${ }^{20}$, also 15 Jahre nach der Vertreibung des Ordens aus Deutschland durch Napoleon und deren Sanktionierung durch den Wiener Kongress. Weber stellt über Tannenberg fest: Der Tag von Tannenberg sollte der letzte Tag des Ordens seyn! Doch Der deutsche Orden erlosch mit Ehren! Und dann zog der weltläufige Weber Vergleiche: Mit diesem unglücklichen Tage, der viel der Römerschlacht an der Trebia, bei Trasimene und Cannaegleicht, noch mehraber derSchlacht vo n Jen a 1806 - erlosch der Glanz des Ordens auf immer! Hier mag das tiefe Bedauern eines Schriftstellers mitschwingen, der eine enge persönliche Verbindung zum Orden gehabt hatte, doch der Vergleich zeigt bereits in eine Richtung, die in der abschließenden Wertung der Ordensgeschichte noch deutlicher aufgegriffen wird: Erging unter in der R eformation, um im schwarzen Adler - dem Sinnbilde der Hochmeister - fortzuleben, und unter demsch warzen eisernen Kreuz - dem Symbol der Ritter - schöner aufzublühen! Preußen blühe, so lange die Ordens-Devise gewahret wird: Suum Cuique! ${ }^{21}$ Die preußische Traditionsaneignung, symbolisch im Eisernen Kreuz dokumentiert, wurde auch von dem Franken Weber akzeptiert, obwohl Tannenberg für ihn das Symbol des Untergangs darstellte.

Neben dem Verschweigen von 1410 steht als zweites, wichtigeres Bild das Darstellungspaar Tannenberg und Heinrich von Plauen, so dass die Entwicklung sich zwar als ein langdauerndes Untergangsszenario darbietet, aber: Der deutsche Orden erlosch mit Ehren! ${ }^{22}$ Diese Umformung zum Heldentum gipfelte schließlich 1881 in Ernst Wicherts Roman Heinrich von Plauen. ${ }^{23}$ Ein später Nachklang ist in Wer-

18 Vgl. M. Blümcke, Karl Julius Weber, der Demokrit aus Hohenlohe (Marbacher Magazin 70), Marbach/Neckar 1996.

19 Vgl. K. Oldenhage, Kurfürst Erzherzog Maximilian Franz als Hoch- und Deutschmeister (1780-1801) (Quellen und Studien zur Geschichte des Deutschen Ordens 34), Bad Godesberg 1969, S. $73 \mathrm{f}$.

20 Hier benutzt C. J. Weber, Das Ritter-Wesen und die Templer, Johanniter und Marianer oder DeutschOrdens-Ritter insbesondere, Bd. 3 (Carl Julius Weber's sämmtliche Werke 14), Stuttgart ${ }^{2} 1837$; die folgenden Zitate dort S. 151-153.

21 Ebd., S. 156.

22 Ebd., S. 153.

23 E. Wichert, Heinrich von Plauen, Leipzig 1881. 
ner Peiners Entwurf Die Belagerung der Marienburg 1410 für eine Tapisserie der geplanten neuen Reichskanzlei für Adolf Hitler 1939 zu sehen (Abb. 3). ${ }^{24}$ In beiden Reaktionsformen ist jedoch ein symbolischer Vorgang erkennbar: das negative Symbol der Scham im Verschweigen, das trotzige Aufbegehren im Heldenkult als Reaktion auf jene Scham.

Erst im 20. Jahrhundert gelang die Umkehr Tannenbergs in ein positives Symbol. 1914 erfocht die deutsche 8. Armee unter Paul von Hindenburg und seinem Generalstabschef Erich Ludendorff über die russische »Narewarmee « in Ostpreußen einen bedeutenden Sieg. Der Kaiser gratulierte zum Sieg bei Allenstein, die Armeeberichte sprachen von der Schlacht in der Gegend von Ortelsburg - Gilgenburg. Der 1. Generalstabsoffizier Ludendorffs, Max Hoffmann, berichtete 1926: General Ludendorff fing beim Diktieren des Befehls damit an: »Frögenau, Zeit offen lassen «. Ich machte ihn darauf aufmerksam, ob er nicht anstatt Frögenau den historischen Namen des vor uns liegenden Ortes Tannenberg wäblen wollte, was er denn auch tat und was später dazu führte, die Schlacht nach dem Ort zu benennen, von dem der Abschlußbefebl des Oberkommandos datiert war. ${ }^{25}$ Ludendorff hatte bereits 1919 in seinen Kriegserinnerungen globaler geschrieben: Die Schlacht wurde auf meinen Vorschlag die Schlacht von Tannenberg benannt, als Erinnerung an jenen Kampf, in dem der Deutsche Ritterorden den vereinigten litauischen und polnischen Armeen unterlag. ${ }^{26}$ So galt die zweite Schlacht von Tannenberg als eine späte Rache des Deutschen Ordens, auch wenn man die Russen des Jahres 1914 mit den Polen und Litauern des Jahres 1410 gleichsetzen musste. Sven Ekdahl hat dazu mit Recht gesagt: „Die Symbolik blieb schief “. 27 Trotzdem wurde der Name Tannenberg zum positiven Symbol in Deutschland, das weitergeführt wurde im Tannenbergdenkmal, welches mit dem Grab Hindenburgs zum Ehrenmal des nationalsozialistischen Deutschen Reiches umfunktioniert wurde $\left(\right.$ Abb. 4). ${ }^{28}$

Nach dem Zweiten Weltkrieg war das Thema Tannenberg in der Deutschen Demokratischen Republik uninteressant und wurde Polen überlassen. In der Bundesrepublik Deutschland lag auch kein Grund zu positiver Symbolik mehr vor, hatte

24 800 Jahre (wie Anm. 4), S. 492 f. mit Farbabbildung VII.4.30.

25 M. Hoffmann, Tannenberg, wie es wirklich war, Berlin 1926, S. 73.

26 E. Ludendorff, Meine Kriegserinnerungen, Berlin 1919, S. 44.

27 Ekdahl (wie Anm. 10), S. 288.

28 Als Beispiel die Veröffentlichung des Kuratoriums für das Reichsehrenmal: Tannenberg. Deutsches Schicksal - Deutsche Aufgabe, Oldenburg i. O. [1939], mit einer „Kundgebung“" von Adolf Hitler. Vgl. auch E. Vogelsang, Aus der Geschichte des Reichsehrenmals Tannenberg, in: Zwischen den Weltkriegen, T1. II: Kultur im Preußenland der Jahre 1918 bis 1939, hrsg. v. U. Arnold (Tagungsberichte der Historischen Kommission für ost- und westpreußische Landesforschung 7), Lüneburg 1987, S. 73-122; J. Tietz, Das Tannenberg-Nationaldenkmal. Architektur, Geschichte, Kontext, Berlin 1999. Abbildung: http://de.academic.ru/dic.nsf/dewiki/1169262. 
doch der Anfall der ehemals deutschen Ostgebiete an Polen jener Symbolik im wahrsten Sinne des Wortes den Boden entzogen. Das Thema Tannenberg spielte nur noch eine Rolle in Vertriebenenkreisen und als Abwehr gegen polnische Benutzung jener Geschichte als politisches Symbol oder aber bei Angriffen aus linkskatholischen Kreisen gegen den gegenwärtigen Deutschen Orden. ${ }^{29}$

Diese allgemein marginalisierende Entwicklung für das Thema Tannenberg wird wieder deutlich durch einen Blick in (bundes-)deutsche Schulbücher. ${ }^{30}$ Ein weit verbreitetes Werk war die Reise in die Vergangenheit - Tannenberg kommt nicht vor. ${ }^{31}$ Die 1975 auch im Blick auf die damals laufenden Schulbuchgespräche zwischen der Bundesrepublik Deutschland und der Volksrepublik Polen neu konzipierte Geschichtliche Weltkunde schreibt unter der Kapitelüberschrift „Polen wird Großmacht": Bald standen sich Ordensstaat und Jagiellonenreich feindlich gegenüber; aus einzelnen Streitigkeiten entwickelte sich ein Krieg, bei dem die Ordensritter in der entscheidenden Schlacht bei Tannenberg 1410 unterlagen..$^{32}$ Das gleichzeitig entwickelte Quellen- und Arbeitsbuch Fragen an die Geschichte subsumiert den Deutschen Orden unter das Kapitel „Die mittelalterliche Ostsiedlung “, wobei Tannenberg nicht vorkommt. ${ }^{33} 1985$ erschien ein bayerisches Schulbuch, das die Ordensgeschichte unter das Generalthema „Slawen und Deutsche“ stellte. Tannenberg wird erwähnt, nüchtern als Folge von 1309 (Erwerb Danzigs und Pommerellens) und 1386 (Vereinigung Polens und Litauens). ${ }^{34}$ Drei Jahre später erschien ein neues Buch, Entdecken und Verstehen. Auch hier findet Tannenberg Erwähnung als Folge von 1386, wieder unter dem Generalthema „Die deutsche Ostsiedlung “. ${ }^{35}$ Nach der Lehrplanrevision zu Beginn der 90er Jahre erschien Geschichte und Geschehen. Auch dieses Buch

29 Vgl. z. B. Contra-Punkte oder die Kunst sich zu irren, hrsg. v. K. Wieser, Wien 1968.

30 Hier kann nur eine kleine Auswahl berücksichtigt werden, da in Deutschland jedes der 16 Bundesländer eine eigene Kultusbehörde hat, die über die Zulassung von Schulbüchern entscheidet, so dass eine Vielzahl von Büchern existiert. Ebenso wird keine Rücksicht genommen auf die unterschiedlichen Schulformen (Hauptschule, Realschule, Gymnasium, Gesamtschule), da in allen Schulformen das Thema in derselben Altersstufe behandelt wird. Allerdings sind nicht alle Bücher auch für alle Schulformen zugelassen, da für jede Schulform eine gesonderte Zulassung vorgenommen wird.

31 H. Ebeling, W. Birkenfeld, Die Reise in die Vergangenheit, Ausgabe N, Band 1: Von der Vorgeschichte bis zum Ende des Mittelalters, Braunschweig 1977, S. 133.

32 W. Hug, H. Busley, Geschichtliche Weltkunde, Bd. 1: Von der frühen Zeit der Menschen bis zum Beginn der Neuzeit, Frankfurt/Main 1974, S. 188.

33 Fragen an die Geschichte. Geschichtliches Arbeitsbuch für Sekundarstufe I, Bd. 2: Die europäische Christenheit, hrsg. v. H. D. Schmid, Frankfurt/Main, hier benutzt ${ }^{5} 1980$, S. 139-149.

34 J. Cornelissen u. a., bsv Geschichte 2 N: Vom frühen Mittelalter bis zum Zeitalter des Absolutismus, München 1985, S. 116.

35 Entdecken und Verstehen, Bd. 1: Von der Urgeschichte bis zum Mittelalter, hrsg. v. T. Berger u. a., Frankfurt/Main 1988, S. 184. 
erwähnt Tannenberg, als Folge von 1386. ${ }^{36}$ Eine Neuedition desselben Titels aus dem Jahre 2008, stärker als Arbeitsbuch gestaltet, widmet sich ausführlicher der preußischen Deutschordensgeschichte einschließlich der Rezeption im 19. und 20. Jahrhundert; Tannenberg ist als Niederlage des Ordens erwähnt, die sich in ein allgemeines Niedergangsszenario einordnet. ${ }^{37}$ Deutlich wird daran, dass die Schulbücher das Thema wenn überhaupt, dann sehr knapp und sachlich darstellen, jedoch keinerlei Ansatzpunkte für politische Positionen mehr bieten.

Tannenberg und Grunwald waren in Preußen/Deutschland wie in Polen Symbole, wenngleich von unterschiedlicher Art und unterschiedlichem Gewicht. In Deutschland überwog lange Zeit der Schamfaktor mit dem Versuch des Verschweigens oder der Reaktion, Niederlage und Heldentum unmittelbar miteinander zu verbinden, so dass das Heldentum die Niederlage überstrahlen konnte. Erst die Masurenschlacht von 1914 vermochte Tannenberg zu einem positiven Symbol zu machen, wenn auch in Schieflage durch eine weitgreifende, panslawische Identifikation. Jedoch nie war Tannenberg so populär, dass es Eingang im Sprichwort gefunden hätte. Man erlebte sein Waterloo, nie jedoch sein Tannenberg. Und nach 1945 spielte es keine wesentliche politische Rolle mehr, tauchte allenfalls als sachliches Datum in Schulbüchern oder anderen Geschichtsdarstellungen auf. Innerhalb der Europäischen Union benötigt Deutschland wie auch Polen den Mythos Tannenberg/ /Grunwald nicht mehr. Die bisherige polnische Gleichsetzung des historischen Deutschen Ordens mit Preußen/Deutschem Kaiserreich/Weimarer Republik/ /„Drittem Reich“/Bundesrepublik Deutschland gehört der Vergangenheit an, wie die Einladung des polnischen Staatspräsidenten an den Hochmeister des heutigen Deutschen Ordens in Wien zur Teilnahme an der Gedenkfeier in Grunwald 2010 zeigt. Seine Ansprache am 15. Juli war in Polen ein mediales Ereignis (Abb. 5) ${ }^{38}$, in Deutschland ist sie nicht zur Kenntnis genommen worden - die deutschen Medien interessierten sich nur für die anschließenden Ritterspiele. Dies ist vielleicht der beste Beleg dafür, dass die ältere deutsche Tradition von Tannenberg erloschen ist.

\footnotetext{
36 G. Birk u. a., Geschichte und Geschehen A 2, Stuttgart 1995, S. 157.

37 F. Behne u. a., Geschichte und Geschehen, Stuttgart 2008, S. 191.

38 Druck in: Tannenberg/Grunwald 2010. Gesammelte Beiträge (Miscellanea Ordinis Teutonici 1), Wien 2010, S. 63-65, Abb. 15-17.
} 
AbBILDUNGeN

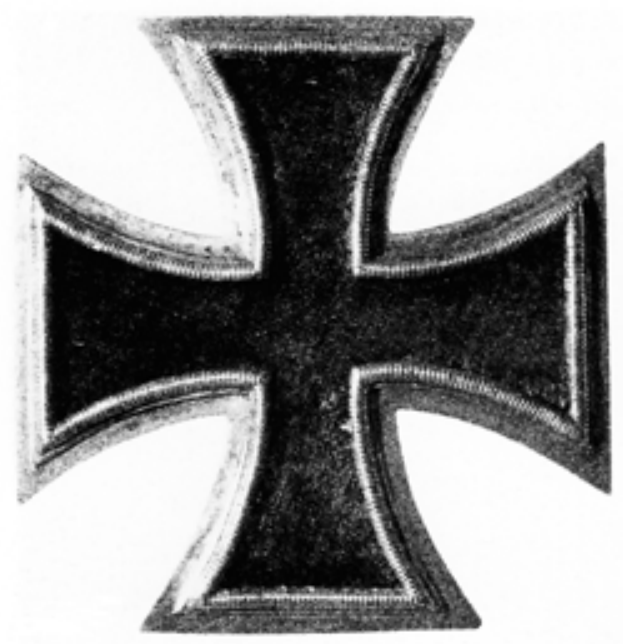

Abb. 1

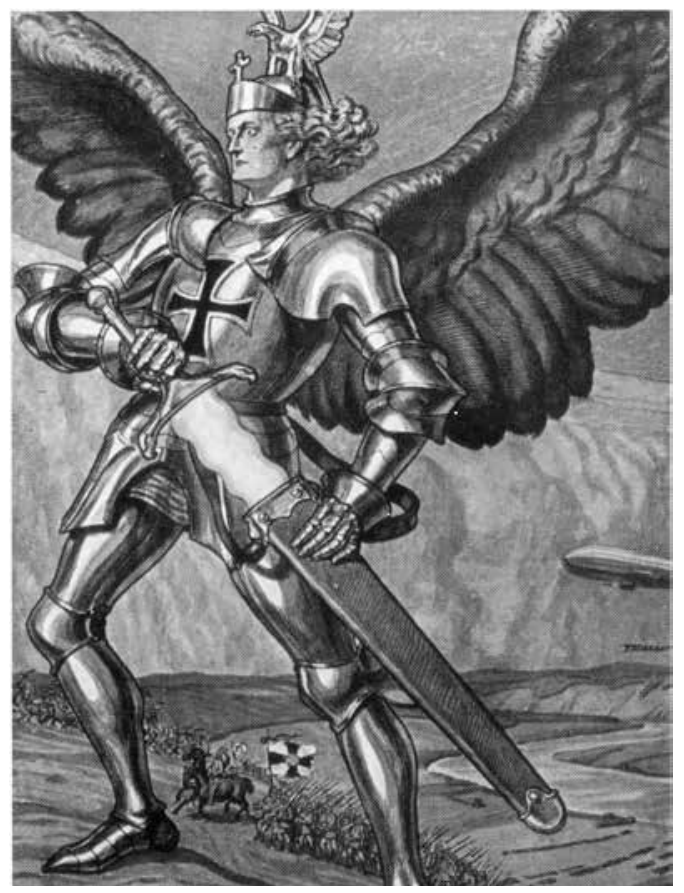

Abb. 2 


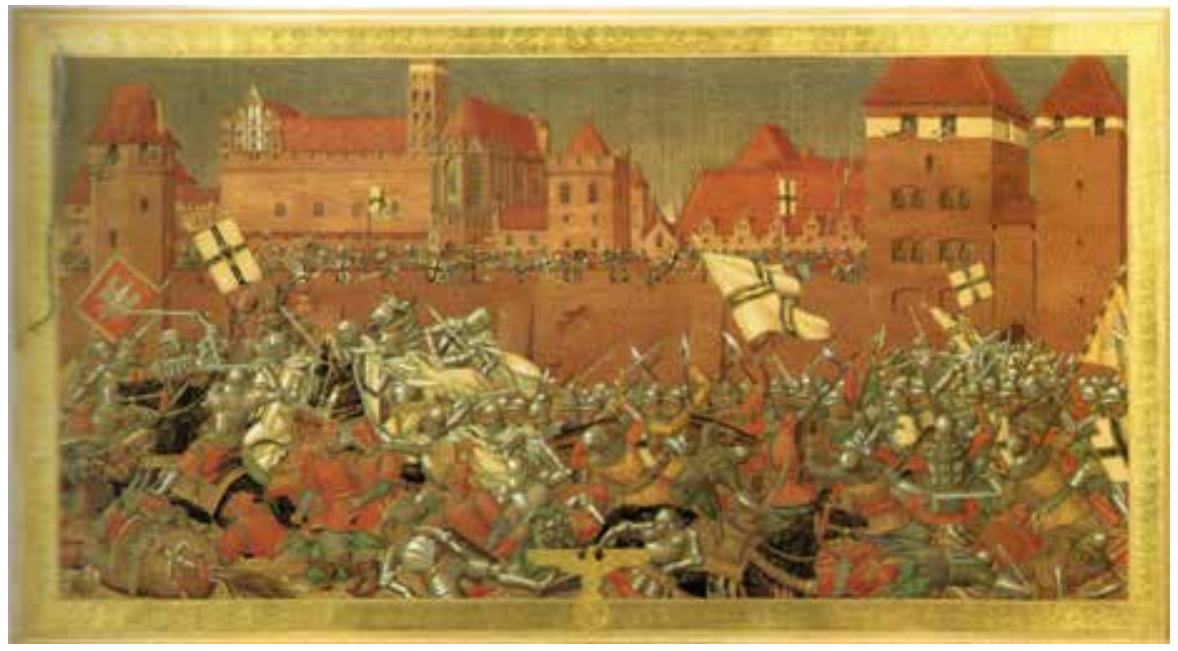

Abb. 3

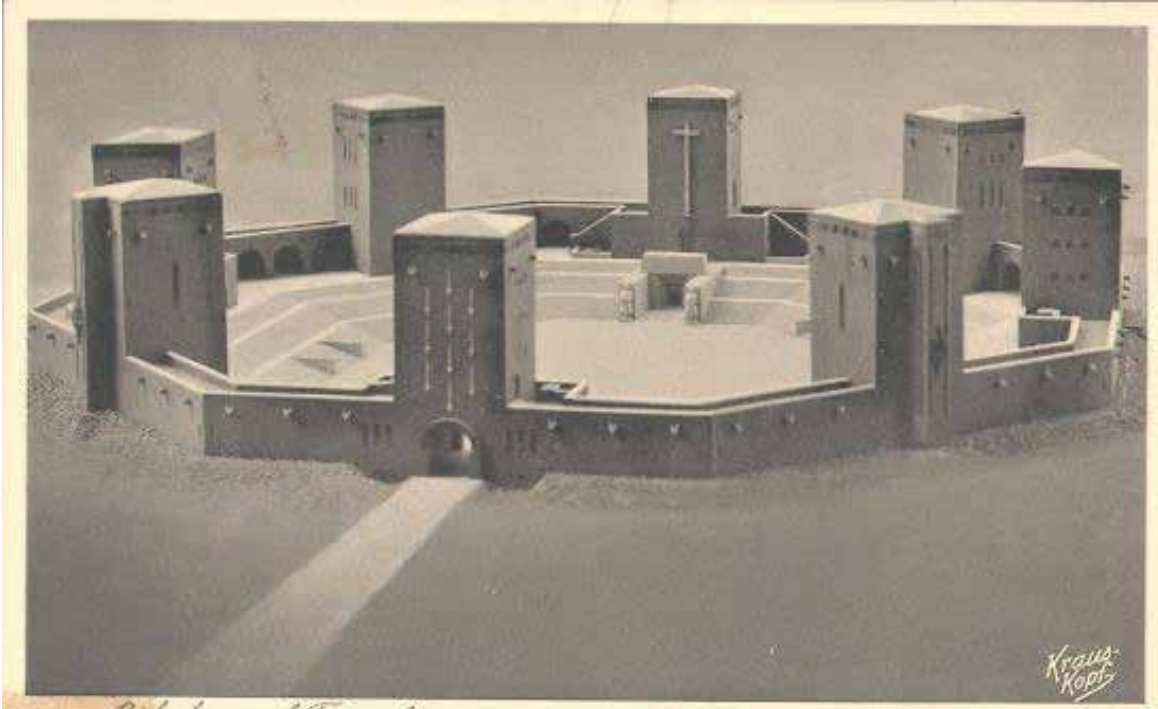

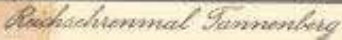

Abb. 4 


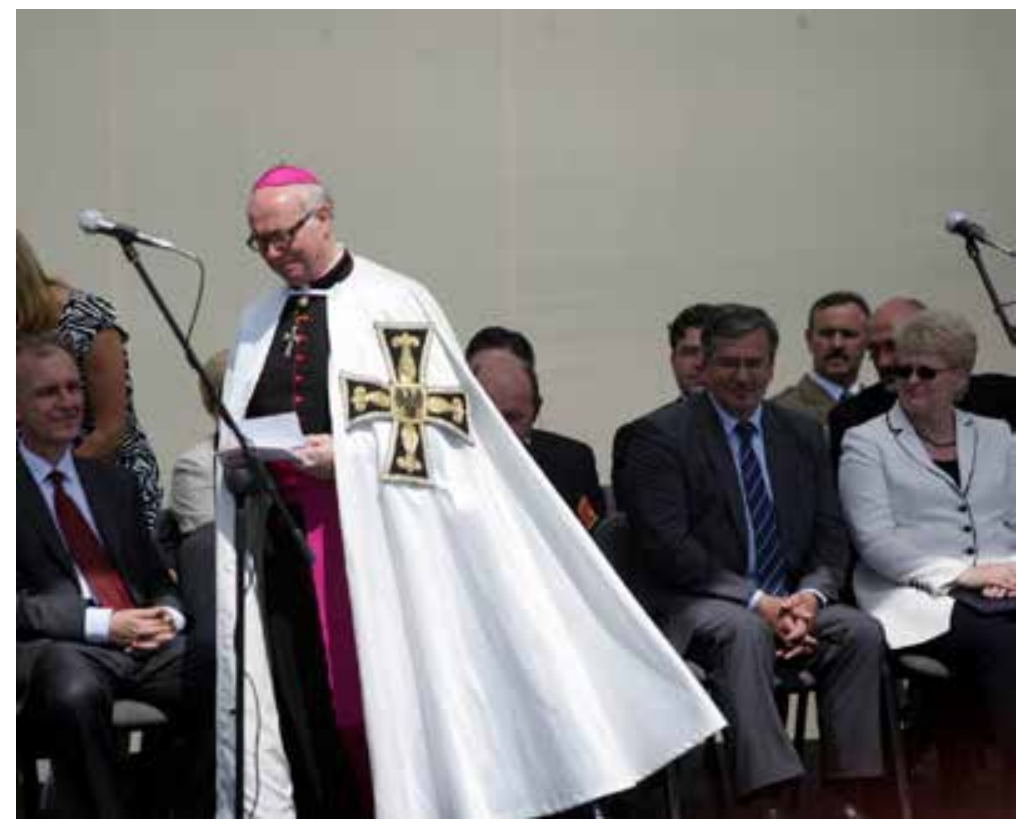

Abb. 5

FOTONACHWEIS

Abb. 1: 800 Jahre (wie Anm. 4), S. 448.

Abb. 2: 800 Jahre (wie Anm. 4), S. 475.

Abb. 3: 800 Jahre (wie Anm. 4), Farbabb. VII.4.30.

Abb. 4: http://de.academic.ru/dic.nsf/dewiki/1169262 (10. 1. 2015)

Abb. 5: Kancelaria Prezydenta RP: http://www.prezydent.pl/aktualnosci/zdjecia/galeria.407.html (10.1.2015)

\section{SUMmaRY \\ Tannenberg (Grunwald) in the German tadition of the $19^{\text {th }}$ and $20^{\text {th }}$ centuries}

Tannenberg (Grunwald) in the German tradition of the $19^{\text {th }}$ and $20^{\text {th }}$ centuries Throughout early modern history, the battle of Grunwald, or Tannenberg in German, has served as an im- 
portant symbol in both Poland and Germany. From the perspective of the latter, for a long time it evoked a sense of shame and defeat that German historiography tried to conceal by confronting it with the heroism of Heinrich von Plauen and the successful defence of Marienburg after the battle. This was the official narrative promoted in German schools.

Following the German victory over the Russian Army in Tannenberg in 1914, the site was recast as a positive symbol of German might in the east. The Teutonic defeat of 1410 and the victory of 1914 were presented as part o the same struggle with Russians and Poles who were ascribed the same, pan-Slavic identity. Nonetheless, these efforts were only partially successful as the battle of 1914 was not as famous as other major battles such as Waterloo.

After 1945 Tannenberg gradually disappeared from German popular imagination. It was remembered by those expelled from the east as part of their local history but is no longer presented as crucial in Germany history books for schools. Its symbolic value for the German historiography therefore, is largely lost. 
